nghĩa thống kê và tương tự nghiên cứu của Cheatum ${ }^{10}$. Như vậy ghi nhận có tổn thương dạ dày tá tràng ở bệnh nhân viêm khớp dạng thấp có sử dụng glucocorticoid.

\section{KẾT LUẬN}

- 40\% bệnh nhân có triệu chứng lâm sàng da dày tá tràng và $60 \%$ bệnh nhân không có biểu hiện triệu chứng lâm sàng.

- 18,2\% bệnh nhân có tổn thương dạ dày tá tràng trên nội soi. Điểm Lanza sửa đổi trung bình là $0,6 \pm 1,396$.

- Nhóm bệnh nhân có triêu chứng lâm sàng đường tiêu hóa có nguy cơ tổn thương dạ dày tá tràng trên nội soi cao gấp 8,857 lần so với nhóm bệnh nhân không có triệu chứng lâm sàng, sự khác biệt có ý nghĩa thônng kê với $p=0,01$ (OR: 8,857: 1,662-47,196).

\section{TÀI LIÊU THAM KHẢO}

1. Neeck G. Fifty years of experience with cortisone therapy in the study and treatment of rheumatoid arthritis. Annals of the New York Academy of Sciences. 2002;966(1):28-38.

2. Overman RA, Yeh J-Y, Deal CL. Prevalence of oral glucocorticoid usage in the United States: A general population perspective. Arthritis Care \& Research. 2013;65(2):294-298. doi:10.1002/acr.21796

3. Naito $Y$, Yoshikawa $T$, Iinuma $S$, et al. Rebamipide protects against indomethacin-induced gastric mucosal injury in healthy volunteers in a double-blind, placebo-controlled study. Dig Dis Sci. 1998;43(9 Suppl):83S-89S.
4. Nguyến Chí Thành. Nghiên cứu tình trạng sử dụng và hiểu biết của bệnh nhân viêm khớp dạng thấp về thuốc corticoid. Published online 2017.

5. Zhang $Y$, Li $H$, Wu $N$, Dong $X$, Zheng $Y$. Retrospective study of the clinical characteristics and risk factors of rheumatoid arthritis-associated interstitial lung disease. Clin Rheumatol. 2017; 36(4):817-823. doi:10.1007/s10067-017-3561-5

6. Nguyển Thị Ngọc Lan. Nghiên cứu tổn thương da dayy tá tràng ỡ bênh nhân mắc bênh khớp điều trị thuốc chống viềm không Steroid. Published online 2003.

7. da Mota $L M H$, dos Santos Neto $L L$, de Carvalho JF, et al. The presence of anticitrullinated protein antibodies (ACPA) and rheumatoid factor on patients with rheumatoid arthritis (RA) does not interfere with the chance of clinical remission in a follow-up of 3 years. Rheumatol Int. 2012;32(12):3807-3812. doi:10.1007/s00296-011-2260-9

8. Tsujimoto $\mathbf{S}$, Mokuda $\mathbf{S}$, Matoba $\mathbf{K}$, et al. The prevalence of endoscopic gastric mucosal damage in patients with rheumatoid arthritis. Pagnini $C_{1}$ ed. PLOS ONE. 2018;13(7):e0200023. doi:10.1371/ journal.pone.0200023

9. Matsukawa $Y$, Aoki $M$, Nishinarita $S$, et al. Prevalence of Helicobacter pylori in NSAID users with gastric ulcer. Rheumatology. 2003;42(8):947950. doi:10.1093/rheumatology/keg258

10. Cheatum DE, Arvanitakis $C$, Gumpel M, Stead H, Steven Geis G. An endoscopic study of gastroduodenal lesions induced by nonsteroidal anti-inflammatory drugs. Clinical Therapeutics. 1999;21(6):992-1003. doi:10.1016/S0149-2918 (99)80020-4

\title{
SO SÁNH KẾT QUẢ THỤ TINH TRONG ỐNG NGHIỆM TRÊN BÊNH NHÂN ĐÁP ỨNG KÉM GIỮA PHÁC Đồ CHU KỲ TỰ NHIÊN VÀ KÍCH THÍCH BUỒNG TRỨNG NHE
}

\section{TÓM TẮT.}

Đáp ứng kém luôn là thách thức trong thụ tinh ống nghiệm. KTBT nhẹ và CKTN là 2 phác đồ sử dụng cho bệnh nhân đáp ứng kém trong thời gian gần đầy. Muc tiều: Đánh giá kết quả TTTON của 2 phác đồ KTBT nhe và CKTN trên bênh nhân đáp ứng kém. Đối tượng nghiên cứu: 96 chu kì thực hiện IVF (49 chu kỳ dùng CKTN, 47 chu kỳ dùng KTBT nhẹ), tại trung

\footnotetext{
${ }^{1}$ Bệnh viện Hồng Ngọc

2Trường ĐHY Hà Nôi

Chịu trách nhiệm chính: Nguyễn Hồng Hạnh

Email: hanh011087@gmail.com-0347911588

Ngày nhận bài: 16.6.2021

Ngày phản biện khoa học: 16.8.2021

Ngày duyệt bài: 24.8.2021
}

\section{Nguyễn Hồng Hạnh ${ }^{1}$, Hồ Sỹ Hùng ${ }^{2}$}

tâm HTSS bênh viên Hồng Ngoc từ tháng 10/2019 đến tháng 5/2021. Phương pháp nghiên cứu: Nghiên cứu so sánh tiến cứu. Kết quả: Ơ nhóm dùng CKTN: $48,9 \%$ chu kỳ thu được noãn, 38,8\% chu kỳ có noãn thụ tinh, $24,5 \%$ chu kì̀ có phôi chuyển. Tỉ lệ làm tổ $25 \%$, tỉ lê thu tinh $79,2 \%$, tỉ lê thai lâm sàng $6,1 \%$. Ơ nhóm dùng KTBT nhẹ: $76,6 \%$ chu kỳ thu được noãn, $63,8 \%$ chu kỳ có noãn thu tinh, 57,4\% chu kỳ có phôi chuyển. Tỉ lệ làm tổ $18,9 \%$, tỉ lệ thụ tinh $67,1 \%$, tỉ lệ thai lâm sàng 14,9\%. Kết luân: khả năng thu được noãn, số phôi thu được, số phồi chuyển ở nhóm dùng KTBT nhe cao hơn so với nhóm dùng phác đồ CKTN. Tuy nhiên tỷ lệ thụ tinh, tỷ lệ làm tô, tỷ lệ thai lâm sàng khác biệt khồng có ý nghĩa giữa 2 phác đồ và không khác biệt với KTBT thông thường.

Tư khóa: Chu kì tự nhiên, kích thích nhe buông trứng, đáp ứng kém, thụ tinh ống nghiệm. 


\section{SUMMARY}

COMPARE RESULT OF IN VITRO

FERTILIZATION BETWEEN NATURE CYCLE AND MILD STIMULATION IN PATIENTS WITH POOR RESPONDER

Poor responder is always a challenge in IVF. Mild stimulation and natural cycle are 2 regimens used for patients with poor responder in recent time. Objectives: To evaluate the results of 2 protocols in IVF for patients with poor responder. Subjects: 96 cycles (49 cycles with natural cycle, 47 cycles with mild stimulation) at the Hong Ngoc IVF Center from October 2019 to May 2021. Methods: prospective comparative study. Results: In the group using natural cycle: $48,9 \%$ of cycles obtained oocytes, $38,8 \%$ of cycles with 2 PN oocytes, $24,5 \%$ of cycles with transferred embryos. Implantation rate $25 \%$, fertilization rate $79,2 \%$, clinical pregnancy rate $6,1 \%$. In the group using mild stimulation: $76.6 \%$ cycles obtained oocytes, $63.8 \%$ cycles 2PN oocytes, $57.4 \%$ cycles with transferred embryos. Implantation rate $18.9 \%$, fertilization rate $67.1 \%$, clinical pregnancy rate $14.9 \%$. Conclusion: The ability to obtain oocytes, the number of obtained embryos in the group using mild stimulation were higher than those in the group using natural cycle. However, the fertilization rate, implantation rate, clinical pregnancy rate did not differ between the two protocols and did not differ from conventional IVF.

Key words: natural cycle, mild stimulation, poor responder, ivf

\section{I. ĐĂT VẤN ĐỀ}

Đáp ứng kém là tình trạng phụ nữ cần dùng liều thuốc kích thích buồng trứng (KTBT) cao nhưng số lượng noãn ít. Hậu quả là giảm số phôi, giảm tỷ lệ có thai, tăng chi phí điều trị. Có nhiều tiêu chuẩn chẩn đoán và phân loại khác nhau được sử dụng cho nhóm bệnh nhần đáp ứng kém trong đó tiêu chuẩn Bologna ${ }^{1}$ năm 2011 và POSEIDON (2016) được nhiêuu tác giả áp dụng ${ }^{2}$, tiêu chuẩn Poseidon chia các bệnh nhân này ra làm 4 nhóm. Trong đó các bệnh nhân nhóm 3 và 4 chiếm đa số các trường hợp với đặc điểm số nang thứ cấp dưới 5 nang và nồng độ AMH dưới 1,2ng/mL.

Nhiều giải pháp được đưa ra đối với bệnh nhân đáp ứng kém như tăng liều $\mathrm{FSH}$, dùng androgen, kích thích kép... nhưng chưa có hướng điều trị thực sự tối ưư ${ }^{2}$. Gần đây, xu hướng mới cho nhóm bệnh nhân này như sử dung phác đồ chu kì tự nhiên hoăc kích thích buồng trứng nhẹ. mục tiêu: So sánh hiệu quả kích thích buồng trứng trên các bệnh nhân đáp ứng buồng trứng kém giữa hai phác đồ chu kì tự nhiên và kích thích nhẹ buồng trứng.

\section{II. ĐỐI TƯỢNG VÀ PHƯƠNG PHÁP NGHIÊN CỨU}

2.1. Đối tượng và phương pháp nghiên cứu. Các cặp vợ chồng điều trị vô sinh bằng phương pháp thụ tinh trong ống nghiệm tại Bệnh viện Hồng Ngọc, giảm dự trữ buồng trứng với tiều chuấn < 5 nang thứ cấp và nồng độ $\mathrm{AMH}$ dưới $1,2 \mathrm{ng} / \mathrm{mL}$. Các trường hợp cho nhận noãn loại khỏi nghiên cứu này. Các bênh nhân này được chia vào 2 nhóm theo dõi chu kì tự nhiên hoặc kích thích buồng trứng nhẹ.

Các bệnh nhân nhóm chu kì tự nhiên không sử dụng thuốc, được theo dõi từ đầu chu kì cho đến khi có nang trưởng thành $18 \mathrm{~mm}$ thì tiêm 6500 đv hCG tái tổ hợp, và được chọc hút noãn sau 36h. Các bênh nhân nhóm kích thích nhe buồng trứng được sử dụng clomiphen citrat (CC) $100 \mathrm{mg}$ hoặc Femara $5 \mathrm{mg}$ từ ngày thứ 2 chu kì. Tùy vào nang noãn phát triển sẽ được dùng thêm tối đa 150 đv FSH tái tổ hợp cho đển khi có nang trưởng thành $18 \mathrm{~mm}$ sẽ được tiêm 6500 hCG tái tổ hợp và chọc hút noãn sau 36 giờ.

Cả hai nhóm được thực hiên tiêm tinh trùng vào bào tương noãn (ICSI) và chuyển phôi ngày 3, nếu trường hợp niêm mạc tử cung không thuận lợi sẽ đông phôi và chuyển phôi đông lạnh vào chu kì sau. Xét nghiệm $\beta$ hCG vào ngày 14 sau chuyển phôi và siêu ầm sau đó 2 tuần nếu xét nghiệm có thai.

2.2. Thiết kế và cỡ mẫu nghiên cứu: Nghiên cứu tiến cứu so sánh. Các bềnh nhân đủ tiêu chuẩn tiêu chuẩn lựa chọn và loại trừ từ tháng 10/2019 đến tháng 5/2021 được thu nhận vào nghiên cứu và phân vào 2 nhóm nghiên cứu với cỡ mẫu thu được $n=96$, với 49 bênh nhân nhóm chu kì tự nhiên và 47 bệnh nhân nhóm KTBT nhe.

2.3. Phương pháp thống kê và xử lý số liệu: Số liêuu thu thập theo các tiêu chí đã nêu theo mẫu bệnh án. Các số liệu được phân tích và xử lý theo phương pháp thống kê y học bằng máy tính với phần mềm SPSS 20.0 .

\section{KẾT QUẢ NGHIÊN CỨU}

\section{1. Đăc điểm lâm sàng và dự trữ buồng trứng nhóm bệnh nhân nghiên cứu}

Bảng 3.1. Đăc điểm lâm sàng của bệh nhân

\begin{tabular}{|c|c|c|c|}
\hline & Chu kì tự nhiên & KTBT nhẹ & p \\
\hline Tuối & $39,16 \pm 3,95$ & $38,15 \pm 5,88$ & 0,32 \\
\hline BMI & $21,17 \pm 1,78$ & $21,40 \pm 2,62$ & 0,61 \\
\hline Thời gian vô sinh & $5,79 \pm 2,15$ & $5,12 \pm 2,35$ & 0,15 \\
\hline
\end{tabular}




\begin{tabular}{|c|c|c|c|}
\hline FSH cơ bản & $10,24 \pm 2,55$ & $8,85 \pm 3,39$ & 0,02 \\
\hline AMH & $0,54 \pm 0,36$ & $0,68 \pm 0,4$ & 0,07 \\
\hline AFC & $2,86 \pm 1,63$ & $33,38 \pm 1,29$ & 0,08 \\
\hline
\end{tabular}

Nhận xét: Sự khác biệt về tuối, chỉ số BMI, thời gian vô sinh giữa 2 nhóm không có ý nghĩa. Dự trữ buổng trứng giữa hai nhóm khác nhau không có ý nghĩa thể hiện qua nồng độ $A M H$, số nang thứ cấp đầu chu kì (AFC), tuy nhiên nồng độ $\mathrm{FSH}$ cơ bản cao hơn ở nhóm dùng phác đồ chu kì tự nhiên so với nhóm dùng phác đồ kích thích buồng trứng nhẹ $(p=0,02)$.

\subsection{So sánh hiệu quả KTBT giữa phác đồ CKTN và KTBT nhẹ}

Bảng 3.2. Kêt quả KTBT

\begin{tabular}{|c|c|c|c|}
\hline & Chu kì tự nhiên & KTBT nhẹ & $\mathrm{p}$ \\
\hline Thời gian KTBT & $0,00 \pm 0,00$ & $6,80 \pm 2,90$ & 0,00 \\
\hline Tống liều Gonadotropin & $0,00 \pm 0,00$ & $222,87 \pm 222,91$ & 0,00 \\
\hline Số noãn chọc hút được & $0,53 \pm 0,50$ & $1,49 \pm 1,41$ & 0,00 \\
\hline Số noãn trưởng thành & $0,49 \pm 0,50$ & $1,25 \pm 1,03$ & 0,00 \\
\hline Số noãn thụ tinh & $0,43 \pm 0,42$ & $1,02 \pm 0,94$ & 0,00 \\
\hline Số phôi & $0,37 \pm 0,40$ & $0,83 \pm 0,76$ & 0,02 \\
\hline Tỷ lệ hưy chu kỳ $(n, \%)$ & $11(22,5)$ & $6(12,8)$ & 0,21 \\
\hline Tỷ lệ chọc hút không noãn $(n, \%)$ & $14(28,6)$ & $5(10,6)$ & 0,04 \\
\hline
\end{tabular}

Nhận xét: thời gian KTBT và tống liều Gonadotropin sử dụng có sự khác biệt rõ ràng giữa nhóm sử dụng phác đồ KTBT nhe với nhóm chu kì tự nhiên, số noãn thu được, số noãn trưởng thành và số noãn thụ tinh, số phôi trung bình cao hơn ở nhóm KTBT, sự khác biêt có ý nghĩa thống kê với $\mathrm{p}<$ 0,05. Tỷ lệ hủy chu kì cao hơn ở nhóm CKTN nhưng sự khác biệt không có ý nghĩa thống kê với $p=0,21$. Tỷ lệ chọc hút không thu được noãn cao hơn ở nhóm sử dụng phác đồ CKTN với $p=0,04$.

Bảng 3.3. Kêt quả tạo phôi và tý lệ có thai

\begin{tabular}{|c|c|c|c|}
\hline & Chu kì tự nhiên & KTBT nhẹ & p \\
\hline Tỉ lệ thụ tinh $(\%)$ & $19 / 24(79,2)$ & $47 / 70(67,1)$ & 0,32 \\
\hline Tì lệ làm tố $(\%)$ & $3 / 12(25)$ & $7 / 37(18,9)$ & 0,84 \\
\hline Tỷ lệ $\beta$ hCG $(+)$ & $5(10,2)$ & $8(17)$ & 0,32 \\
\hline Thai lầ sàng $(\mathrm{n}, \%)$ & $3(6,1)$ & $7(14,9)$ & 0,19 \\
\hline Thai sinh hóa $(\mathrm{n}, \%)$ & $2(4,1)$ & $1(2,1)$ & 0,58 \\
\hline
\end{tabular}

Nhận xét: Tỉ lệ làm tố và tỉ lệ thụ tinh thai lâm sàng và thai sinh hóa giữa 2 phác đồ khác biệt không có ý nghĩa thống kê.

\section{BÀN LUẬN}

4.1. Đặc điểm lâm sàng và dự trữ buồng trứng của bệnh nhân. Tuổi trung bình nhóm CKTN là $39,16 \pm 3,95$ và nhóm KTBT nhe là $38,15+5,88$ cao nhất 49 tuổi, trẻ nhất là 26 tuổi và tuổi hay gặp là 37 tuổi. Theo kết quả nghiên cứu của Trương Văn Tuyên ${ }^{3}$ (2014) trên bệnh nhân đáp ứng kém bằng phác đồ antagonist thì tuổi trung bình là $34,13 \pm 5,19$. Chỉ số BMI trung bình, thời gian vô sinh trung bình của 2 nhóm khác nhau không có ý nghĩa và đều trong giới hạn bình thường, kết quả này tương tự nghiên cứu của Lê Long Hồ ${ }^{4}$. Thời gian mong con trung bình là $6,70 \pm 5,70$. Theo Zhen ${ }^{8}$ thời gian vô sinh trung bình ở nhóm đáp ứng kém và nhóm đáp ứng bình thường là tương đương $(7,2 \pm 4,9$ và $7,02 \pm 4,8)$. Các đặc điểm lâm sàng của bệnh nhân về độ tuổi trung bình, chỉ số khối cơ thể (BMI), thời gian vô sinh giữa 2 nhóm là không có sự khác biệt. Trong nghiên cứu của chúng tôi tiêu chuẩn lựa chọn bệnh nhân là Poseidon nhóm 3 và 4 nhưng trên thực tế số bệnh nhân Poseidon nhóm 3 ít nên dấn tới độ tuổi trung bình khá cao.

Nồng độ $A M H$ trung bình khác biệt không có ý nghĩa giữa 2 nhóm. Theo nghiên cứu của Lê Long Hồ ${ }^{4}$ thì nồng độ $\mathrm{AMH}$ trung bình ở nhóm Poseidon 3 là $0,7 \pm 0,33$, nhóm Poseidon 4 là $0,57 \pm 0,36$. Giảm dự trữ buồng trứng làm giảm tỷ lệ có thai, đặc biệt ở nhóm Poseidon 3 và 4, việc đánh giá và điều trị trước khi thực hiện IVF/ISCI gặp nhiều khó khăn ${ }^{5,6}$.

Có sự khác biệt về nồng độ $\mathrm{FSH}$ cơ bản giữa 2 nhóm, ở nhóm CKTN nồng độ FSH cơ bản cao hơn $(10,24 \pm 2,55$ với 8,85 $\pm 3,39)$, nồng độ AMH thấp hơn $(0,54 \pm 0,36$ với $0,68 \pm 0,40)$, AFC thấp hơn $(2,86 \pm 1,63$ với $3,38 \pm 1,29)$ so với nhóm sử dụng phác đồ KTBT nhẹ, tuy nhiên sự khác biệt này không có ý nghĩa thống kê. Nghiên cứu của Drakopulous ${ }^{7}$, nồng độ FSH trung bình là 10,2 IU/L ở nhóm sử dụng phác đồ KTBT liều cao và nồng độ FSH trung bình là 12,6 
IU/L ở nhóm sử dụng phác đồ CKTN. Theo nghiên cứu của Zhen (2008), nồng độ cơ bản của nhóm đáp ứng kém là $11,8 \pm 5,1$ cao hơn nhóm bình thường $8,33 \pm 2,89$ có ý nghĩa thống kê $\hat{e}^{8}$. Như vậy nồng độ $\mathrm{FSH}$ cơ bản càng cao thì khả năng đáp ứng kém càng lớn.

4.2. So sánh hiệu quả KTBT giữa 2 phác đồ

4.2.1. Về đặc điểm chu kì điêuu trị và kết quả kích thích buồng trứng. Trong nghiên cứu này nhóm CKTN hoàn toàn không sử dụng $\mathrm{FSH}$, còn nhóm KTBT nhe chỉ dùng tối đa 150 đv/ngày, tổng liều trung bình là $222,87+222,91$ với thời gian KTBT trung bình là $6,8 \pm 2,9$ ngày. So với nghiên cứu của Trương Văn Tuyên ${ }^{3}$ (2014) trên nhóm bệnh nhân đáp ứng kém với phác đồ antagonist là 9,72 $\pm 1,28$ ngày, thì số ngày sử dụng Gonadotropin của chúng tôi ít hơn và tổng liêu là $2705 \pm 1073 đ v$ thì tổng liêu Gonadotropin sử dụng trên mối bệnh nhân thấp rõ rệt trong nghiên cứu của chúng tôi, hơn nữa nhóm CKTN hoàn toàn không KTBT. Việc tiêm thuốc KTBT liều cao và kéo dài làm tăng chi phí cũng như gây căng thẳng và lo lắng cho bệnh nhân.

Sự khác biệt có ý nghĩa thống kê về thời gian kích thích buồng trứng và tổng liêuu Gonadotropin giữa nhóm sử dụng phác đồ CKTN với nhóm sử dụng phác đồ KTBT với $p<0,05$. Do phác đồ CKTN dựa vào sự phát triển sinh lý của nang noãn nển số noãn chọc hút được, số noãn trưởng thành ít hơn so với nhóm sử dụng phác đồ KTBT nhẹ. Sự khác biệt này có ý nghĩa thống kê với $p<0,05$.

Số noãn chọc hút trong nhóm CKTN là $0,53+$ 0,5 và nhóm KTBT nhẹ là $1,49+1,41$, sự khác biệt có ý nghĩa thống kê. Theo nghiên cứu của Drakoupoulus ${ }^{7}$ (2019), khi sử dụng phác đồ CKTN số noãn trung bình chọc hút được là $0,8 \pm$ 0,6 , phổ biến nhất là thu được 1 noãn, có trường hợp không thu được noãn. Cũng theo nghiên cứu này, số noãn trưởng thành trung bình là 0,7 $\pm 0,6$. Điều này cho thấy với phác đồ chu kì tự nhiên số noãn thu được chủ yếu là 1 , có những trường hợp chọc hút không thu được noãn vì đó có thể là nang trống. Đây cũng là phù hợp với sinh lý phát triển nang noãn vì thực tế trong tự nhiên mỗi chu kì cũng chỉ có 1 nang vượt trội.

Trong nghiên cứu của chúng tôi, tỉ lệ hủy chu kì là 11 trường hợp $(22,5 \%)$ ở nhóm chu kì tự nhiên và 6 trường hợp $(12,8 \%)$ ở nhóm KTBT nhe. Sự khác biệt này không có ý nghĩa. Tuy nhiên tî̉ lệ chọc hút không noãn là $28 \%$ ở nhóm CKTN và $10 \%$ ở nhóm KTBT nhe lại khác biệt có ý nghĩa, điều này được giải thích trong chu kì KTBT nhẹ thường có trên 1 nang trong khi trong
CKTN thường chỉ có 1 nang phát triển nên khả năng chọc hút không có noãn rất dễ xảy ra. Việc sử dụng phác đồ chu kì tự nhiên là gần như hoàn toàn dựa vào chu kì sinh lý của mỗi bệnh nhân không có bất kì tác động nào trên sự phát triển của nang noãn nên hiện tượng có những chu kì nang noãn không phát triển hoặc phóng noãn trước chọc là điều có thể xảy ra. Việc xác định chính xác thời điểm chọc hút trứng để tránh hiện tượng phóng noãn trước chọc là rất quan trọng nhằm giảm thiểu tỉ lệ hủy chu kì không đáng có.

Dựa trên kết quả trên nghiên cứu của chúng tôi cùng nghiên cứu của một số tác giả khác có thể thấy trên nhóm bệnh nhân đáp ứng kém theo phân loại Bologna hay phân loại Poseidon thì sử dụng phác đồ KTBT nhẹ tối ưu hơn ở sô noãn thu được, số noãn MII đồng thời giảm tỷ lệ hủy chu kì.

Một trong những điểm đáng chú ý trong nghiên cứu của chúng tôi là tỷ lệ chọc hút không noãn cao ở nhóm bệnh nhân sử dụng phác đồ CKTN (28,6\%). Do nhóm bệnh nhân trong nghiên cứu chủ yếu là bệnh nhân lớn tuổi, $\mathrm{AMH}$ thấp, đáp ứng buồng trứng kém. Những bệnh nhân chọc hút không thu được noãn phần lớn là bệnh nhân từ 40 tuổi trở lên. Ở phụ nữ lớn tuổi, số lượng và chất lượng noãn giảm nhanh vì thế có nhiều chu kì chỉ có nang trống. Thực tế trên lâm sàng, trong các chu kì chọc hút noãn có hiện tượng được ghi nhận là hội chứng nang trống (EFS- Empty follicle syndrome). Nguyên nhân của hội chứng này do tuổi của người bệnh (tỷ lệ EFS lên đến $57 \%$ với bệnh nhân trên 40 tuổi), khả năng đáp ứng kém và $A M H$ thấp.

4.2.2. Kêt quá tạo phôi và tỷ lệ có thai. Tỉ lệ thụ tinh ở nhóm dùng phác đồ CKTN cao hơn ở nhóm dùng phác đồ KTBT nhẹ nhưng sự khác biệt không có ý nghĩa thống kể. Điều này cho thấy ở phác đồ CKTN số lượng noãn thu được ít hơn so với KTBT nhẹ, nhưng khả năng thụ tinh tốt hơn. Hầu hết noãn thu được đều thụ tinh $(79,2 \%)$ trong khi đó KTBT nhe dù thu được noãn nhiêu hơn nhưng tỉ lệ thụ tinh thấp hởn $(67,2 \%)$. Điều này cho thấy chất lượng noãn có xu hướng tốt hơn khi chọn lọc nang noãn theo tự nhiên không có tác động ngoại sinh. Nghiên cứu của chúng tôi tập trung đối tượng đáp ứng kém phần lớn là phụ nữ lớn tuổi, $A M H$ thấp.

Số phôi thu được ở nhóm KTBT nhẹ cao hơn nhóm CKTN $(0,83 \pm 0,76$ với $0,37 \pm 0,40)$ sự khác biệt có ý nghĩa thống kê với $p<0,05$.

Tỉ lể làm tổ ở nhóm CKTN cao hơn nhóm KTBT nhẹ (25\% so với $18,9 \%)$ nhưng sự khác 
biệt không có ý nghĩa thống kê.

Thực tế trong nghiên cứu của chúng tôi, tỷ lệ thai lâm sàng trên tổng số bệnh nhân là $6,1 \%$ ở CKTN và $14,9 \%$ ở KTBT nhe. Tỉ lẹ thai lâm sàng ở nhóm dùng phác đồ KTBT nhẹ cao hơn so với nhóm dùng phác đồ CKTN. Tướng tự tỷ lệ thai sinh hóa nhóm dùng phác đồ KTBT nhe thấp hơn so với nhóm dùng phác đồ CKTN. Tuy nhiên cả 2 sự khác biệt này không có ý nghĩa thống kê với $p>0,05$.

\section{KẾT LUÂN}

Qua kết quả nghiên cứu này chúng tôi nhận thây khả năng thu được noãn, số noãn chọc hút được, số phôi tạo thành ở nhóm KTBT nhẹ cao hơn so với nhóm dùng phác đồ CKTN. Tuy nhiên tỷ lệ thụ tinh, tỷ lệ làm tổ, tỷ lệ thai lâm sàng không có sự khác biệt giữa 2 phác đồ và không khác biệt với KTBT thông thường. Nghiên cứu nhằm mục đích giúp bác sĩ lâm sàng có thêm lựa chọn điều trị cho bệnh nhân đáp ứng kém khi đứng trước cân nhắc chi phí và hiệu quả điều trị.

\section{TÀI LIÊU THAM KHẢO}

1. Ferraretti A.P, La Marca. A, Fauser.B.C.J.M, et al. (2011). ESHRE consensus on the definition of 'poor response' to ovarian stimulation for in vitro fertilization: the Bologna criteria". Human Reproduction. 26(7):pp.1616-1624.

2. Alviggi $C_{\text {, Andersen }} \mathrm{CY}$, Buehler $\mathrm{K}$, Conforti
A, De Placido G, Estevas SC, et al (2016). A new more detailed stratification of low resonders to ovarian stimulation: from a poor ovarian response to a low prognosis concept. Fertil Steril; 105(6): 1452-3.

3. Trương Văn Tuyên (2014), Nghiên cứu kết quả thụ tinh trong ông nghiệm trên bệnh nhân đáp ứng kém với kích thích buônng trứng bằng phác đô Antagonist tại Bệnh viện phụ sản Trung Ương, Luân văn thac sỹ Y hoc, Đai học Y Hà Nôii, Hà Nôi.

4. Lê Long Hồ (2016). Tỷ lệ thai diễn tiến cộng dồn khi điều trị thu tinh trong ống nghiệm của các bênh nhân tiền lương thấp theo phân loại POSEIDON. Tạp chí y học sinh sản 12, 72-75.

5. Haiquing Tian, Ximin Mao, Nam Su, Xiaolin La (2021). The correlation between $A M H$ and number of embryos in POSEIDON groups: a retrospective cohort study. Reproductive Biomedicine Online 42(4), pp.842-848.

6. M. B. Palhares, R. A. Ferriani, W. P. Martins, P. A. Navarro (2015). A formula combining age, $\mathrm{AMH}, \mathrm{AFC}$ and $\mathrm{FSH}$ is more accurate than individual markers in predicting poor response to cotrolled ovarian stimulation in good prognosis patients. Fertility and Sterility 104(3), p.654.

7. Panagiotis Drakapoulus, Alessia Romito, Christophe Blockeel (2019). Modified natural cycle IVF versus conventional stimulation in advanced-age Bologna poor responders. Reproductive Biomedicine Online 39(4), pp.698-703.

8. Zhen X.M., Quiao J., Li R., Wang L.N., Liu P. (2008). The clinical analysis of poor ovarian response in in-vitro-fertilization embryo-transfer among Chinese couples. J Assist Reprod Genet, 25(1), pp.17-22.

\section{ĐĂC ĐIỂM TỔN THƯƠNG MẮT Ở BỆNH NHÂN VIÊM CộT SỐNG DÍNH KHỚP}

\section{TÓM TẮT}

Mục tiêu nghiên cứu: Mô tả đặc điểm tổn thương mắt ở bệnh nhân viêm cột sống dính khớp. Đối tượng nghiên cứu: 32 bệnh nhân được chẩn đoán viểm cột sống dính khớp theo tiêu chuẩn New York sửa đổi 1984, điều trị nội trú tại khoa Cơ xương khớp, bệnh viện Bạch Mai từ tháng 12/2020 đến tháng 4/2021. Phướng pháp nghiên cứu: Nghiên cứu mô tả cắt ngang kết hợp hồi cứu bệnh án. Kết quả: Tỷ lệ bệnh nhân có triệu chứng cơ năng ở mắt là $18,7 \%$, trong đó triêu chứng nhìn mờ chiếm tỷ lê $15,6 \%$; đỏ mắt 3,1\%; có $81,3 \%$ bênh nhân không có triệu chứng cơ năng tại mắt. Tỷ lệ bệnh nhân có tổn thướng thực

${ }^{1}$ Trường đại học Y Hà Nội.

${ }^{2}$ Bênh viên Bach Mai.

Chịu trách nhiệm chính: Nguyễn Văn Hiển

Email: hiennv789@gmail.com

Ngày nhận bài: 14.6.2021

Ngày phản biên khoa hoc: 12.8.2021

Ngày duyệt bài: 20.8.2021 thể tai mắt là $25 \%$, trong đó viêm màng bô đào chiếm tỷ lệ 21,9\%; viêm kết mạc 3,1\%; có 75\% bệnh nhân không có tổn thương thực thể tại mắt. Kết luận: Cần khám mắt ở bệnh nhân viêm cột sống dính khớp do ít biểu hiện triệu chứng, tuy viêm màng bồ đào có tỷ lệ thấp nhưng có thể gây mất thị lực không hồi phục nếu không đước phát hiên và điều tri kip thời.

Tư khóa: Viêm cột sống dính khớp, tổn thương mắt, viêm màng bồ đào.

\section{SUMMARY \\ CHARACTERISTICS OF OCULAR INVOLVEMENT IN ANKYLOSING SPONDYLITIS PATIENTS}

Ojective: To decribe the characteristics of ocular involvements in patients with ankylosing spondylitis. Subjects: 32 patients with ankylosing spondylitis were diagnosed using Modified New York Criteria 1984, in the department of Rheumatology, Bach Mai hospital from December 2020 to April 2021. Methods: Retrospective and cross-sectional descriptive study. Results: The proportion of patients 